\title{
Research modular tool system influence on the dynamic characteristics of the boring machines
}

\author{
Alexey Rivkin ${ }^{1, *}$ \\ ${ }^{1}$ Moscow State University of Technology “STANKIN” Vadkovsky lane 3a, Moscow 127055, Russia
}

\begin{abstract}
The article presents the analysis of modern modular tooling systems (MTS). The calculations of the stiffness of joints modules compounds "Yartim-Flex» and dynamic research of spindles of machine tools models IR-320PMF30 and 2D450F2, equipped with this modular tool system.
\end{abstract}

The most important component of modern computer-integrated production is the process control system. Since rational technology is, first of all, the effective use of the tool, the organization of the technological system of any production is the main and decisive organization and functioning of the subsystem of tool support [1, 2].

Among the numerous requirements for the cutting tool, when using CNC machines, the following should be highlighted:

1. Versatility of application for typical machined surfaces of different parts on different models of machines with a limited range of tools.

2. Quick change when changing to another workpiece or replacing blunted tool;

3. The ability to adjust the size outside the machine (in conjunction with the auxiliary tool used).

These requirements do not allow in a number of cases to use in flexible production systems (FPS) cutting tools used on General-purpose machines, and currently for CNC machines (as part of FPS) allocated a special group of complete cutting tools, and some of it is already standardized.

Taking into account the features of CNC machines designed for processing body parts, the shanks of the auxiliary tool contain elements that ensure its fixation in the spindle, and for machines with automatic tool change-additional elements that allow you to capture it by the operator for transportation from the store to the machine spindle and back $[3,4]$.

In order to increase the versatility of the tool and at the same time simplify the design, block-modular tool systems for CNC machines of different types have been developed, which provide a great effect thanks to the group technology in tool production.

Composed of standardized structural modules cutting tools are readjusted in relation to the change of the workpiece by a simple rearrangement of standardized interchangeable units.

To compile tools of different types use a certain number of parts and components (modules), which form an interconnected structure that has sufficient resulting stiffness and accuracy. This design allows you to create combined tools, change their lengths and

* Corresponding author: alexey-rivkin@yandex.ru 
diameters in accordance with each specific processing task. Therefore, the block-modular design principle allows you to order not just a tool of a certain type, but a tool system with the necessary replaceable devices.

Currently, to pair the individual blocks of the modular tool with each other and install the tool in the spindle of the machine, various types of connecting elements are used, which must ensure high rigidity and accuracy of the connection. All these elements can be divided into two groups: connecting elements with rigid links and connecting elements with elastic links.

The most common connecting elements with rigid links is a standard mandrel with taper 7:24, links with a short taper and a flat end face (Novex, Walter, Sandvik Coromant), the connection cylinder and the planar end face (Graflex, Varilok Sandvik Coromant, Hertel, Schmalcalden, Dornag, Polledzi, CO, Krupp Widia, Komet, Mapal,Tizit and others.)

Due to the deviations of the profiles of the connecting surfaces of the chain links from the ideal, their contact is not over the entire surface, but only on several points, which strongly reduces the stiffness of the connection. The discrepancy between the angles of the cones inner and outer holes particularly strong influence on the stiffness of the conical connections. The most unfavorable is the case when the taper angle of the hole is greater than the mandrel, as it seems to lengthen the cantilever part of the mandrel [5].

The solution to the problem can be the use of elastic links, which are deformed, reduce the impact of manufacturing errors, thereby increasing the contact area and stiffness of the connection.

The connecting elements with elastic links are a base end and a cylindrical or conical collet, which centers the links relative to each other and, working together with the end, increases the stiffness of the connection. Fundamentally these compounds differ from each other only way fastening, which affects effort tightening [6].

Double basing in such constructions is eliminated due to the fact that

1. The rigidity of the transition place from the end surface to the cylindrical or conical is reduced due to the annular inner recess

2. Centering on the cylindrical or conical surface of the joint occurs after basing on the end surface.

For release based cylinder use either the internal activity collets (Güring, Aachen, Mapal), or the distribution of the balls (Valenite, Widaflex, Krupp Vidia) or a screw with a conical thread (Sandvik Coromant, Novex NC, Walter, ARTEM-Flex) (Fig. 1).

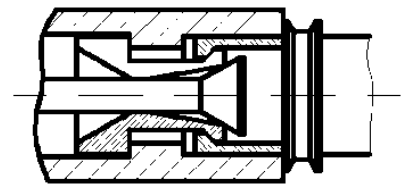

a)

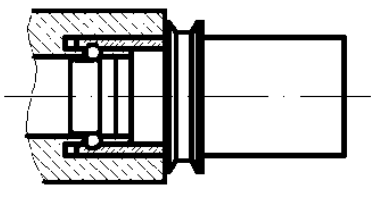

b)

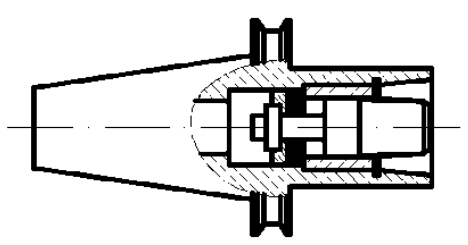

c)

Fig. 1. The ways of connection of modules of modular tool systems:

a) using the collet; b) using the unclenched balls; c) with conical thread

Torque transmission is carried out either by means of end dowels, or by manufacturing a base cylindrical surface in the form of a RK-profile (Sandvik Coromant)

Connection scheme of the YARTIM-Flex system junction is represented in Fig. 2. The collet of the module 4 is inserted into the conical hole of the module 1 with a gap $\Delta=1.2 \ldots 2$ $\mathrm{mm}$; the end of the stud 3 sets the modules relative to each other. 


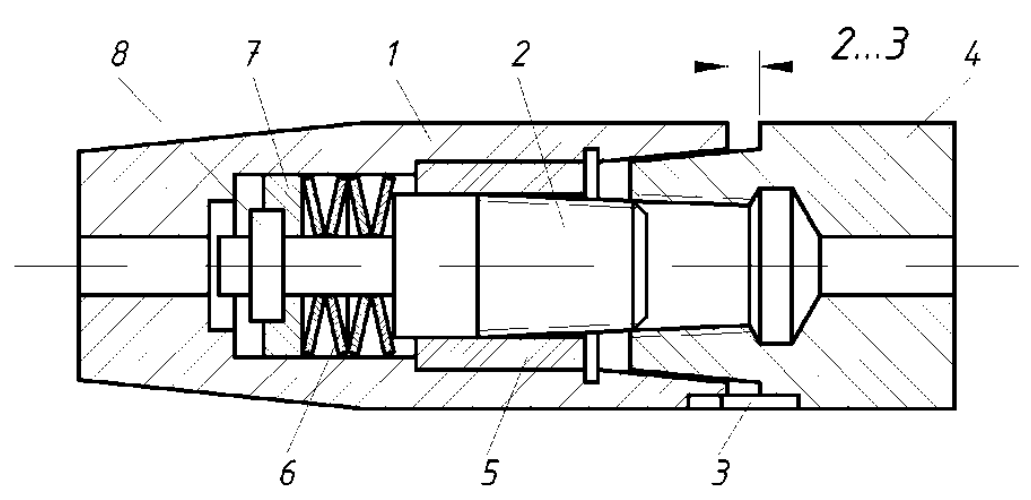

Fig. 2. The scheme of the connection system YARTIM-Flex

By rotating the tightening wrench counterclockwise, the left-hand tapered screw 2 is screwed into the tapered thread of module 4 and compresses the spring layering 6 . Springs 6 , resting on the end of the sleeve 5 , create a force $Q$, which is transmitted by links 7 and 8 to the threaded connection, shifting the module 4 to the contact of the conical surfaces of modules 1 and 4 . At this point, the modules are centered, but there is a gap $\Delta=0.2 \ldots 0.3 \mathrm{~mm}$ between the base surfaces.

With further screwing of the screw 2, the force $Q$ increases and the module 4 moves to the full selection of the gap, thus creating pressure on the end and conical surfaces of the module 4.

When the torque on the screw 2 will reach $80 \mathrm{~N} \cdot \mathrm{m}$, the gap across the contact surface of conical screw thread 2 and the module 4 is selected, the collet module 4 will turn the radial deformation, selecting the gaps along the reference surfaces of the modules 1 and 4 .

The calculation of the stiffness of the joints was carried out according to the method given in [5]. The calculation formula was derived [7], which allowed to obtain the initial data for modeling the elastic system in the basic package of the subroutine SPINCH [8]:

$$
j=\frac{\pi}{16 c} \frac{\sqrt{\pi \cdot Q\left(D^{4}-d^{4}\right)\left(D^{2}+d^{2}\right)}}{16 c}+\frac{d^{2} L \pi}{800} \sqrt{\frac{d E}{2 k}} \frac{1}{\frac{40 L}{d} \sqrt[4]{\frac{0,5 d}{E k} C_{3}}+C_{4}}, N m / r a d
$$

where $j$ is the angular stiffness of the joint; $Q$ - tightening force, $N$; $D$ is the outer diameter of boring bars, $\mathrm{mm}$; $d$ - diameter of the collet, $\mathrm{mm}$; $L$ - length of boring bars, $\mathrm{mm}$; $c$ - dimensional factor, $N^{1 / 2} \mathrm{~mm}^{2}$ [5]; $k$ - stiffness of the joint, $\mathrm{mm} / \mathrm{MPa}$ [5]; $E$ - modulus of elasticity, $M P a$; $C_{3}, C_{4}$ are empirical dimensionless coefficients [5].

To simulate the error of processing it is necessary to make a mathematical model of the spindle unit of the machine, equipped with tool adjustment. As an example, select the spindle assembly of machines mod. IR320PMF4 and 2D450F2, a design scheme is presented in Fig.3.

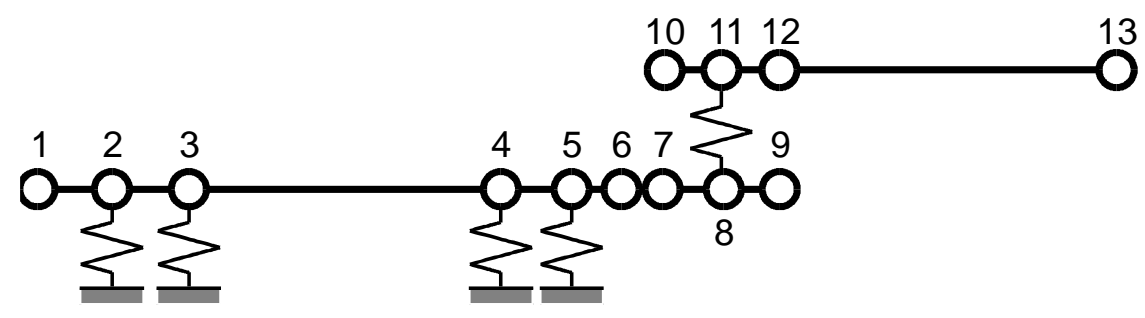

Fig. 3. A design scheme for spindle assembly 
The most convenient way of modeling is the finite element method (FEM). To compile a resolving system of equations in a dynamic formulation, we use the Lagrange equation:

$$
\begin{gathered}
\frac{d}{d t}\left(\frac{\partial T}{\partial \dot{q}_{l}}\right)-\frac{\partial T}{\partial q_{i}}=Q_{i} \\
(i=1,2, \ldots, n),
\end{gathered}
$$

where $t$ is time; $q_{i}$ is the generalized coordinates; $n$ is the number of degrees of freedom; $T$ is the kinetic energy of the system, $Q_{\mathrm{i}}$ is the generalized forces.

After performing the transformations we obtain a system of equations:

$$
[A]\{\ddot{q}\}+[C]\{q\}=[0]
$$

where $[A]$ is the mass matrix, $[C]$ is the stiffness matrix.

Equation (2) describes the free oscillations of the system without attenuation. To describe the forced oscillations with attenuation, it is necessary to add the term $[B]\{\dot{q}\}$ to the left part of the system, where $[B]$ is the damping matrix, and in the right part, instead of the zero matrix, put the matrix of forces acting on the system.

According to [16] the damping matrix can be obtained by replacing in the mass matrix [A] the values of the masses of the elements $a_{\mathrm{ij}}$ by the damping coefficients $b_{\mathrm{ij}}$.

Thus, we obtain a system of differential equations in matrix form:

$$
[A]\{\ddot{q}\}+[B][\dot{q}]+[C]\{q\}=[D]
$$

where $[D]$ is the vector of forces acting on the system.

$\mathrm{n}$ equation (4) in the stiffness matrix [C] there is a stiffness of the conical spindle connection with the tool, the value of which has a great influence on the overall stiffness of the spindle-tool system.

The rigidity of the conical joint, determined by the contact stiffness of the mating surfaces, largely depends on the accuracy of the joint [16].

Fig. 4 shows the frequency response of the spindle units of the above-mentioned machines, obtained as a result of modeling, where the solid line refers to the characteristic of the whole tool, the dotted line to the characteristic of the modular tool. Quality indicators of the subsystem of tool support (SIS) are summarized in table. 1.

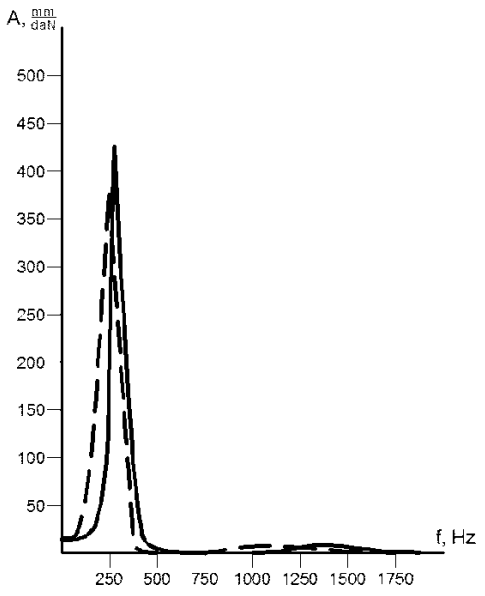

a)

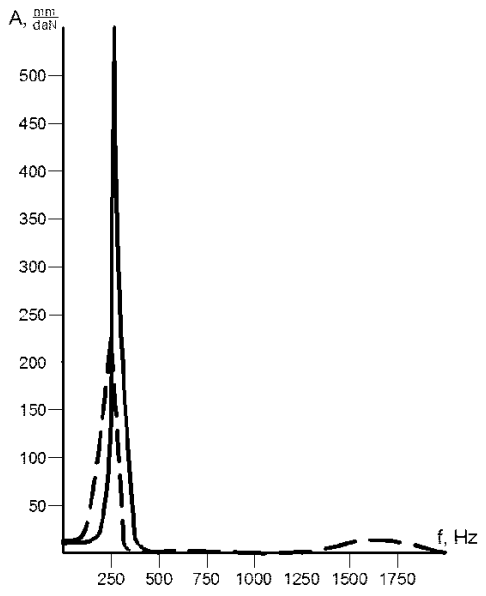

b)

Fig. 4. Frequency response of the spindle assemblies for machine tools IR320PMF4 (a) and 2D450F2 (b) with inserted tool holder 
The results showed:

1. The presence of tooling significantly changes the statics and dynamics of the boring machines spindle assemblies.

2. The traditional one-piece and modular tool setups are completely interchangeable in terms of static and dynamic characteristics.

3. Modular tool has significant advantages associated with versatility, flexibility and manufacturability. The economic effect of their use is due to a decrease in the number of special tools in precision machines coordinate boring group.

Thus, the use of modularity in the system "YARTIM-Flex" is an effective means of reducing the cost of processing while maintaining high precision machining on CNC boring machines.

Perhaps a more detailed study of the spindle units of these machines and similar machines using the planned computer experiment. On the basis of the empirical models obtained, it is proposed to carry out multi-criteria optimization of the basic designs of spindle assemblies of boring machines, taking into account the influence of the drive mechanism and modular tool setups produced by the most famous companies.

Table 1. Comparison of quality indicators of spindle units of machines with different types of tool support systems.

\begin{tabular}{|c|c|c|c|}
\hline \multirow{2}{*}{ Tool's type } & \multirow{2}{*}{$\begin{array}{c}\text { Machine's } \\
\text { model }\end{array}$} & $\begin{array}{c}\text { Quality indicators (determined at a radial load of } 10 \mathrm{~N}, \\
\text { acting at the end of the mandrel with a flight of } 300 \mathrm{~mm} \text { ) }\end{array}$ \\
\cline { 3 - 4 } & & Static deflection, $\mu \mathrm{m}$ & Basic resonance frequency, $\mathrm{Hz}$ \\
\hline One-piece & \multirow{2}{*}{ 2D450F2 } & 52.8 & 275 \\
\cline { 1 - 3 } Modular & & 62.5 & 248 \\
\hline One-piece & \multirow{2}{*}{ IR320PMF4 } & 42.3 & 267 \\
\cline { 1 - 3 } Modular & & 51.8 & 250 \\
\hline
\end{tabular}

\section{References}

1. R.I. Gjyrov, V.A. Grechishnikov, V.G. Logachev, Tool systems of automated production (Polytechnic S.Pt, 1993) (in Russian)

2. V.A. Grechishnikov, V.B. Romanov, P.M. Pivkin Technical creativity of youth, 3(97), pp. 27-29 (2016) (in Russian)

3. A.Y. Nekrasov, M.O. Arbuzov, A.N. Sobolev, Russian Engineering Research, Automatic control of the static loads in multicontact mechanical systems, v. 35(6), pp 442-446 (2015)

4. 4 F.S. Sabirov, L.G. Vainer, A.V. Rivkin, Russian Engineering Research, Vibroacoustic diagnostics of bidirectional end milling, v. 35(6), pp 458-461 (2015)

5. Z.M. Levina, D.N. Reshetov Contact stiffness of machines (Mashinostroenie, Moscow, 1971) (in Russian)

6. O.I. Averianov, V.T. Zheltyakov, Y.A. Pavlov, The creation of modular tooling systems for advanced processing technology, precise holes in a variety of industries (NPF Dynamic, Yaroslavl, 1996) (in Russian)

7. A.V. Rivkin, A.N. Sobolev, A.J Nekrasov, J.A. Pavlov Engineering technology, 8, pp. 19-23 (2017) (in Russian)

8. V.S. Khomyakov, S.I. Dosko, I.V. Bradis Automated system for calculating static and dynamic characteristics of machines: Software. User manual (Mosstankin, Moscow, 1990) (in Russian) 